\title{
Association of Apolipoprotein E Polymorphisms and Risks of Ischemic Stroke in Chinese Patients with Type 2 Diabetes Mellitus
}

\author{
Na Wang $\mathbb{D},{ }^{1}$ Qian Liu, ${ }^{2}$ Hui Liu, ${ }^{2}$ Xiao Cong, ${ }^{2}$ Hui Yang, ${ }^{2}$ Yang Yu, $^{2}$ Yongtong Cao $\mathbb{D},{ }^{1,2}$ \\ and Liang $M a \mathbb{D}^{2}$ \\ ${ }^{1}$ Department of Transfusion Medicine, China-Japan Friendship Hospital, Beijing 100029, China \\ ${ }^{2}$ Clinical Laboratory, China-Japan Friendship Hospital, Beijing 100029, China \\ Correspondence should be addressed to Yongtong Cao; caoyongtong92@sina.com and Liang Ma; liangma321@aliyun.com
}

Received 22 September 2020; Revised 5 December 2020; Accepted 24 December 2020; Published 4 January 2021

Academic Editor: Zhao Zhao

Copyright @ $2021 \mathrm{Na}$ Wang et al. This is an open access article distributed under the Creative Commons Attribution License, which permits unrestricted use, distribution, and reproduction in any medium, provided the original work is properly cited.

\begin{abstract}
Background. The apolipoprotein E (APOE) gene polymorphisms have been intensively studied in patients with type 2 diabetes mellitus (T2DM) and ischemic stroke (IS) in recent years. However, it is unclear whether APOE gene polymorphisms are correlated with increased risk for developing IS in T2DM patients. Thus, this study was designed to examine the association between APOE gene polymorphisms and risks of IS in Chinese patients with T2DM. Methods. This case-control study enrolled 243 subjects with T2DM as controls, and 210 subjects with T2DM complicated with IS as case patients. The genotypes were determined using real-time PCR while HbAlc and lipid levels were detected using commercially available kits. Results. The systolic blood pressure (SBP), diastolic blood pressure (DBP), and the proportion of patients with a history of hypertension were higher in the case patients than that in the controls. We confirmed that the $\varepsilon 2 / \varepsilon 3$ genotype, as well as SBP and history of hypertension, was the independent risk factor for developing IS in T2DM patients. Conclusions. We conclude that the $\varepsilon 2 / \varepsilon 3$ genotype might contribute to the increased risk for developing IS in Chinese patients with T2DM.
\end{abstract}

\section{Introduction}

China has the highest number of diabetes in the world. According to the International Diabetes Federation (IDF) report, there were 116 million Chinese diagnosed with diabetes in 2019, and the number will increase to 141 million by 2030 [1]. Ischemic stroke (IS) is a cerebrovascular complication of type 2 diabetes mellitus (T2DM) due to accelerated atherosclerosis and carotid artery disease development [2]. Both macrovascular and microvascular systems of the brain are severely affected in T2DM [3]. T2DM exacerbates ischemic brain injury and worsens functional outcome after stroke [4]. Patients with T2DM have a two- to four-fold increase in the risk of IS compared with the general population $[5,6]$. Moreover, stroke patients with diabetes have a poor prognosis after stroke onset [7]. Thus, it is of great importance to investigate the risk factors of T2DM patients complicated with IS.
Mounting evidence indicates that the apolipoprotein $\mathrm{E}$ (APOE) gene located at chromosome $19 \mathrm{q} 13.32$ is a candidate gene in the development of T2DM and IS [8-10]. The protein encoded by the APOE gene consists of 299 amino acid residues and contains amphipathic $\alpha$-helical lipid-binding structural domains which enable APOE to interact with members of the low-density lipoprotein receptor family. Through the interaction, APOE plays an essential role in lipid transportation in both plasma and brain [11]. The dysregulation of the $A P O E$ expression and genetic variance of the $A P O E$ influence the APOE functions and lead to the pathogenesis of nervous and cardiovascular diseases eventually [11-13]. The common gene variants, epsilon-2 ( 22$)$, epsilon-3 $(\varepsilon 3)$, and epsilon-4 $(\varepsilon 4)$, are generated by the two single-nucleotide polymorphisms (SNPs) rs7412 (C/T) and rs429358 (C/T) in exon 4 of the $A P O E$ gene. The variants are different haplotypes of the APOE gene generated by the combination of the two SNPs at the APOE locus [14]. The most common allele iso- 
form is $\varepsilon 3$ with a frequency of $70-80 \%$. The $\varepsilon 2$ and $\varepsilon 4$ have a frequency of $5-10 \%$ and $10-15 \%$, respectively [15]. The 3 alleles of the APOE gene form 6 genotypes $(\varepsilon 2 / \varepsilon 2, \varepsilon 2 / \varepsilon 3$, $\varepsilon 2 / \varepsilon 4, \varepsilon 3 / \varepsilon 3, \varepsilon 3 / \varepsilon 4, \varepsilon 4 / \varepsilon 4)$ [16]. The $\varepsilon 3 / \varepsilon 3$ is the most common genotype with a frequency of approximately $67 \%$ [8]. The frequencies of $\varepsilon 3 / \varepsilon 4$ and $\varepsilon 2 / \varepsilon 3$ are lower than those of $\varepsilon 3 / \varepsilon 3$, and $\varepsilon 2 / \varepsilon 2, \varepsilon 4 / \varepsilon 4$, and $\varepsilon 2 / \varepsilon 4$ genotypes have the lowest frequencies [17].

Understanding the risk factors of T2DM patients complicated with IS may shed light into intervening the development of complications of T2DM. So far, studies on the association of APOE polymorphisms and IS risks in Chinese patients with T2DM are lacking. Thus, this study is designed to elucidate whether the $A P O E$ gene polymorphism is an essential determinant of Chinese patients with T2DM complicated with IS.

\section{Materials and Methods}

2.1. Subjects. A case-control study was carried out from July 2015 to July 2018. Informed consent was obtained from all enrolled subjects, and the design protocol was approved by the Ethics Committee of China-Japan Friendship Hospital. The data of the families, their medical history, and smoking habits of the patients was obtained by a questionnaire. Clinical examination including measurement of systolic blood pressure (SBP) and diastolic blood pressure (DBP) was applied. Anthropometric data (weight and height) were collected and used for BMI calculation. Hypertension was defined as blood pressure above $140 / 90 \mathrm{mmHg}$ or taking antihypertensive drugs. Dyslipidemia was characterized by increased total cholesterol (TC $\geq 6.20 \mathrm{mmol} / \mathrm{L}$ ), low-density lipoprotein cholesterol ( $\mathrm{LDL}-\mathrm{C}>4.13 \mathrm{mmol} / \mathrm{L}$ ), and triglyceride (TG $>2.25 \mathrm{mmol} / \mathrm{L}$ ), or decreased high-density lipoprotein cholesterol (HDL $-\mathrm{C}<1.03 \mathrm{mmol} / \mathrm{L})$ [18]. These subjects were categorized into two groups: T2DM patients complicated with and without IS according to the criteria of the American Diabetes Association Classification 2010 [19].

(1) T2DM patients without IS (control group). This group consists of 243 subjects fulfilling the T2DM diagnostic criteria or under diabetes medication (oral and/or insulin) with no history or signs of any IS.

(2) T2DM patients complicated with IS (IS group). This group consists of 210 subjects diagnosed to have T2DM or under diabetes medication and complicated with IS. All the subjects in this group were examined by a qualified neurologist. The diagnosis of IS was confirmed by clinical symptoms or signs, laboratory results, and computed tomography (CT) or magnetic resonance imaging (MRI). Exclusion criteria included cardiac diseases, renal diseases, hepatic diseases, endocrine diseases, metabolic disorders, autoimmune diseases, skeletal disorders, and cancerous diseases.

2.2. DNA Isolation and APOE Genotyping. Blood samples were collected in vacuum tubes and stored at $-20^{\circ} \mathrm{C}$ until processed. DNA was extracted from each blood sample using the genomic DNA purification kit (Xi'an Tianlong Science and Technology Co., Ltd., Xi'an, Shaanxi, China). The concentration of DNA was quantified using a NanoDrop 1000 spectrophotometer (Thermo Fisher Scientific, Waltham, MA, USA). Three APOE alleles $(\varepsilon 2, \varepsilon 3$, and $\varepsilon 4)$ were detected by an $A P O E$ Genotyping Kit (Sinochips Bioscience Co., Ltd., Zhuhai, Guangdong, China). Polymorphic alleles were identified by the fluorescence intensity of the hybridization sites.

2.3. Biochemical Analysis. Plasma levels of fasting TG, TC, LDL-C, and HDL-C were measured using the AU5800 automated biochemical analyzer (Beckman Coulter, Brea, CA, USA). HbA1c was detected using the D-10 Hemoglobin Testing System (Bio-Rad, Hercules, CA, USA).

2.4. Statistical Analysis. The data were statistically analyzed using SPSS version 24.0 software (IBM, Chicago, IL, USA). Quantitative data were expressed as mean values \pm standard deviation (SD). Normally distributed data were compared using Student's $t$-test. The significance of differences in the proportion of patients with a history of hypertension between the two groups was tested by the chi-square test $\left(\chi^{2}\right)$. The differences in the distribution frequencies of genotype and allele between the two groups, and the deviations from Hardy-Weinberg equilibrium were tested by the chisquare test. The extremely rare genotype groups $-\varepsilon 2 / \varepsilon 2$, $\varepsilon 2 / \varepsilon 4$, and $\varepsilon 4 / \varepsilon 4$ - were excluded from the genotype and allele analyses. Univariable logistic regression analysis was used to test the association between IS and APOE gene polymorphisms, and the analysis results were presented as unadjusted odds ratios (OR) with confidence intervals (95\% CI). Multivariate logistic regression was used to determine the risk factors for developing IS in T2DM patients with adjustment for potential covariates: age, gender, BMI, blood pressure, duration of T2DM, smoking, HbA1c level, and plasma lipids, and the results were presented as adjusted ORs. $P<0.05$ was considered to be statistically significant.

\section{Results}

3.1. General Characteristics and Biochemical Variables of the Patients. 210 T2DM patients complicated with IS and 243 age- and sex-matched controls from the same demographic area were included in this study. Table 1 presents the general characteristics and biochemical variables of the patients in the control and the IS groups. No statistically significant differences were observed between the groups in age, gender, BMI, smoking, diabetes duration, HbAlc level, and plasma lipid level $(P>0.05)$. However, SBP, DBP, and the proportion of patients with a history of hypertension were higher in the IS group than in the control group $(P<0.05)$.

3.2. APOE Genotype and Allele Distribution Frequencies in the IS and the Control Groups. The APOE genotype distribution in the IS and the control groups were in HardyWeinberg equilibrium $(P>0.05)$. Distribution frequencies of the 6 genotypes and 3 alleles of $A P O E$ in the IS and the control groups are summarized in Table 2. 
TABle 1: Demographic, clinical, and biochemical data of the IS and the control groups.

\begin{tabular}{lccc}
\hline & IS $(n=210)$ & $\begin{array}{c}\text { Control } \\
(n=243)\end{array}$ & $P$ value \\
\hline Age (years) & $65.41 \pm 8.20$ & $64.58 \pm 10.21$ & 0.338 \\
Gender (male \%) & 47.62 & 52.26 & 0.324 \\
BMI $\left(\mathrm{kg} / \mathrm{mm}^{2}\right)$ & $25.29 \pm 3.98$ & $25.75 \pm 3.19$ & 0.175 \\
SBP $(\mathrm{mmHg})$ & $142.67 \pm 19.41$ & $133.98 \pm 18.63$ & $<0.001$ \\
DBP (mmHg) & $81.64 \pm 10.17$ & $78.17 \pm 10.67$ & $<0.001$ \\
Hypertension (\%) & 84.28 & 70.20 & $<0.001$ \\
Smokers (\%) & 18.57 & 17.70 & 0.809 \\
Diabetes duration & $14.44 \pm 6.09$ & $15.10 \pm 8.32$ & 0.332 \\
(years) & $3.65 \pm 2.10$ & - & - \\
IS duration (years) & $7.66 \pm 1.72$ & $7.96 \pm 1.88$ & 0.084 \\
HbA1c (\%) & $60.25 \pm 18.81$ & $63.45 \pm 20.59$ & 0.084 \\
HbA1c (mmol/mol) & $1.88 \pm 1.62$ & $2.00 \pm 1.46$ & 0.433 \\
TG (mmol/L) & $4.20 \pm 1.24$ & $4.24 \pm 1.14$ & 0.697 \\
TC (mmol/L) & $2.48 \pm 0.95$ & $2.52 \pm 0.99$ & 0.666 \\
LDL-C (mmol/L) & $1.05 \pm 0.33$ & $1.04 \pm 0.31$ & 0.880 \\
HDL-C (mmol/L) & 64.29 & 64.20 & 0.984 \\
Dyslipidemia (\%) & & &
\end{tabular}

3.3. Association of APOE Gene Polymorphisms and IS. The univariate analysis was used to evaluate the association from the perspective of the genotype and allele frequencies of $A P O E$ gene polymorphisms. The genotype of $\varepsilon 2 / \varepsilon 3$ increased the risk of IS in T2DM patients, with unadjusted OR 1.901 (95\% CI $1.117-3.297, P=0.0179)$, while the genotype of $\varepsilon 3 / \varepsilon 4$ showed no association with IS in T2DM patients, with unadjusted OR 1.629 (95\% CI $0.943-2.756, P=0.0694)$ (Table 3). The results displayed no significant difference in $A P O E$ gene allele frequencies between the two groups (Table 3). After being adjusted for age, gender, BMI, SBP, DBP, history of hypertension, smoking habits, diabetes duration, HbAlc, TG, TC, LDL-C, HDL-C, and history of dyslipidemia using multivariable binary logistic regression analysis as shown in Table 4 , the $\varepsilon 2 / \varepsilon 3$ genotype was an independent risk factor for developing IS in T2DM patients, with adjusted OR 2.225 (95\% CI 1.244-3.980, $P=0.007$ ). However, the genotype of $\varepsilon 3 / \varepsilon 4$ was not found to be an independent risk factor for developing IS in T2DM patients, with adjusted OR 1.727 (95\% CI 0.978-3.049, $P=0.060$ ) (Table 4). SBP (95\% CI 1.004-1.031, $P=0.013$ ) and history of hypertension (95\% CI 1.089-3.045, $P=0.022$ ) were also independent risk factors for developing IS in T2DM patients (Table 4).

\section{Discussion}

Many studies showed that patients suffering from T2DM are at a higher risk for IS than individuals without T2DM $[4,20]$. Thus, it is essential to explore the predisposing risk factors for IS among T2DM patients. We carried out a case-control study to investigate the association of the $A P O E$ gene polymorphisms and risks of IS in Chinese patients with T2DM.
We observed that SBP, DBP, and the proportion of patients with a history of hypertension were higher in the IS group than in the control group. We also found that the $\varepsilon 2 / \varepsilon 3$ genotype was an independent risk factor for developing IS in T2DM patients. SBP and history of hypertension were also independent risk factors for developing IS in T2DM patients after adjusting for age, gender, BMI, SBP, DBP, history of hypertension, smoking habits, diabetes duration, HbAlc, TG, TC, LDL-C, HDL-C, and history of dyslipidemia.

A prospective population-based study, with approximately 20-year follow-up, evaluated the effect of T2DM on cardiovascular disease in 13105 samples. The study revealed an increased relative risk for developing stroke of 1.5 to 6.5 fold in T2DM individuals [20]. The increased risk is seen even after early diagnosis. It is reported that the risk of stroke in newly treated patients with T2DM is $9.1 \%$ within the first 5 years. The stroke rate is double that of the general population [21]. Many studies have indicated that T2DM patients have residual neurological deficits and a worse functional outcome, along with worse long-term mortality [7, 22]. Therefore, patients and physicians need to aggressively control cardiovascular risk factors soon after T2DM diagnosis. The identification of susceptibility genes and other risk factors would be helpful for the management of IS in T2DM patients.

$A P O E$ gene is one of the most widely studied candidate genes of T2DM. In a case-control study recruiting of 451 Thais, it has been demonstrated that $\varepsilon 4$ allele containing genotypes were the predictors of T2DM [23]. A metaanalysis of 30 studies including 5423 case patients and 8197 controls suggests that $\varepsilon 2$ allele is associated with increased risk of T2DM [8]. Besides, the relation between APOE gene polymorphisms and IS has been intensively investigated in many studies. Most findings suggest that $\varepsilon 4$ allele increases the odds of IS [24-26]. However, little is known about the link between $A P O E$ gene polymorphisms and developing IS in T2DM patients. So far, there is only one study conducted on the issue. The study displayed that $A P O E$ gene polymorphisms were not linked with IS in T2DM patients [27]. However, we found that T2DM patients who carried the $\varepsilon 2 / \varepsilon 3$ genotype were at 1.90 -fold increased risk to develop IS in the present study. After adjustment for other established risk factors, $\varepsilon 2 / \varepsilon 3$ was an independent risk factor for developing IS in T2DM patients. The discrepancies of the findings may be due to ethnic differences, sample sizes, genotyping method, and other risk factors. APOE gene polymorphisms affect plasma lipid concentration and may be responsible for the development of IS onset in T2DM patients. Further investigation is needed to study the APOE gene polymorphisms and lipid metabolism in T2DM patients complicated with IS.

Consistent with our results, many studies have shown that T2DM patients with hypertension tend to have a higher risk of getting IS $[2,28,29]$. We found that SBP, DBP, and the proportion of patients with a history of hypertension were higher in the IS group compared with that in the control group. Furthermore, SBP and history of hypertension were independent risk factors for developing IS in T2DM patients after adjusting for many related factors. So, proper blood 
TABLE 2: Distribution frequencies of $A P O E$ genotype and allele in the IS and the control groups.

\begin{tabular}{lccccccccc}
\hline & \multicolumn{4}{c}{ APOE genotypes (\%) } & \multicolumn{4}{c}{ APOE alleles (\%) } \\
& $\varepsilon 2 / \varepsilon 2$ & $\varepsilon 2 / \varepsilon 3$ & $\varepsilon 2 / \varepsilon 4$ & $\varepsilon 3 / \varepsilon 3$ & $\varepsilon 3 / \varepsilon 4$ & $\varepsilon 4 / \varepsilon 4$ & $\varepsilon 2$ & $\varepsilon 3$ & $\varepsilon 4$ \\
\hline IS & $2(0.95)$ & $39(18.57)$ & $4(1.90)$ & $126(60.00)$ & $37(17.62)$ & $2(0.95)$ & $47(11.19)$ & $328(78.10)$ & $45(10.71)$ \\
Control & $45(1.65)$ & $28(11.52)$ & $7(2.88)$ & $172(70.78)$ & $31(12.76)$ & $1(0.41)$ & $43(8.85)$ & $403(82.92)$ & $40(8.23)$ \\
\hline
\end{tabular}

TABLE 3: Associations of APOE gene polymorphisms and the risk of IS in T2DM patients compared to that in case patients, represented as unadjusted OR.

\begin{tabular}{lcc}
\hline & Unadjusted OR $(95 \% \mathrm{CI})$ & $P$ value \\
\hline Genotype & & \\
$\varepsilon 2 / \varepsilon 3$ vs. $\varepsilon 3 / \varepsilon 3$ & $1.901(1.117-3.297)$ & 0.0179 \\
$\varepsilon 2 / \varepsilon 3$ vs. $\varepsilon 3 / \varepsilon 4$ & $1.167(0.602-2.281)$ & 0.657 \\
$\varepsilon 3 / \varepsilon 4$ vs. $\varepsilon 3 / \varepsilon 3$ & $1.629(0.943-2.756)$ & 0.0694 \\
Allele & & \\
$\varepsilon 2$ vs. $\varepsilon 3$ & $1.343(0.876-2.073)$ & 0.186 \\
$\varepsilon 2$ vs. $\varepsilon 4$ & $0.972(0.534-1.766)$ & 0.924 \\
$\varepsilon 4$ vs. $\varepsilon 3$ & $1.382(0.882-2.190)$ & 0.157 \\
\hline
\end{tabular}

The extremely rare genotypes- $-\varepsilon 2 / \varepsilon 2, \varepsilon 2 / \varepsilon 4$, and $\varepsilon 4 / \varepsilon 4$ subjects-were excluded from statistical analysis.

TABLE 4: Associations of APOE gene polymorphisms and the risk of IS in T2DM patients compared to that in case patients, represented as adjusted OR.

\begin{tabular}{lcc}
\hline & Adjusted OR $(95 \% \mathrm{CI})$ & $P$ value \\
\hline$\varepsilon 3 / \varepsilon 3$ (referee) & - & 0.010 \\
$\varepsilon 2 / \varepsilon 3$ & $2.225(1.244-3.980)$ & 0.007 \\
$\varepsilon 3 / \varepsilon 4$ & $1.727(0.978-3.049)$ & 0.060 \\
Age & $1.009(0.986-1.033)$ & 0.452 \\
Gender & $0.880(0.570-1.358)$ & 0.563 \\
BMI $\left(\mathrm{kg} / \mathrm{mm}^{2}\right)$ & $0.958(0.901-1.018)$ & 0.166 \\
SBP $(\mathrm{mmHg})$ & $1.017(1.004-1.031)$ & 0.013 \\
DBP $(\mathrm{mmHg})$ & $1.017(0.993-1.042)$ & 0.168 \\
Hypertension $(\%)$ & $1.821(1.089-3.045)$ & 0.022 \\
Smokers $(\%)$ & $1.172(0.672-2.042)$ & 0.576 \\
Diabetes duration & $0.981(0.954-1.009)$ & 0.186 \\
HbA1c & $0.896(0.798-1.006)$ & 0.063 \\
TG & $0.925(0.772-1.109)$ & 0.398 \\
TC & $1.032(0.580-1.835)$ & 0.916 \\
LDL-C & $0.921(0.496-1.710)$ & 0.793 \\
HDL-C & $0.988(0.387-2.517)$ & 0.979 \\
Dyslipidemia & $1.458(0.841-2.529)$ & 0.179 \\
\hline
\end{tabular}

pressure management is a core issue for mitigating the development of IS in T2DM patients.

There are some limitations in our study which require further investigation. Firstly, the replicability of the data needs to be verified in a larger sample size involving different ethnic groups. Secondly, our study is inadequate in proving the causal relationship but merely an association research due to the study design of case-control. However, our find- ings indeed contribute to risk prediction for IS in T2DM patients. The genotype $\varepsilon 2 / \varepsilon 3$ of $A P O E$ is possibly a genetic predisposition factor for IS in T2DM patients. Furthermore, it is of great significance to control the blood pressure for T2DM patients to avoid IS onset.

\section{Conclusions}

Our study indicates that the APOE gene polymorphisms are associated with the development of IS in T2DM patients. We also identify $\varepsilon 2 / \varepsilon 3$ genotype, SBP, and history of hypertension as independent risk factors in the development of IS in T2DM patients.

This study provides data that may help to improve the ability to identify diabetic individuals at increased risk for IS and improve the clinical management of patients with T2DM.

\section{Data Availability}

The data used to support the findings of this study are available from the corresponding authors upon request.

\section{Conflicts of Interest}

The authors declared no conflict of interest.

\section{Acknowledgments}

This work was funded by the National Natural Science Foundation of China (grant number 81703892). We appreciate the involvement of all the doctors, researchers, and patients in this study.

\section{References}

[1] P. Saeedi, I. Petersohn, P. Salpea et al., "Global and regional diabetes prevalence estimates for 2019 and projections for 2030 and 2045: results from the International Diabetes Federation Diabetes Atlas, 9th edition," Diabetes Research and Clinical Practice, vol. 157, p. 107843, 2019.

[2] A. Ergul, A. Kelly-Cobbs, M. Abdalla, and S. C. Fagan, "Cerebrovascular complications of diabetes: focus on stroke," Endocrine, Metabolic \& Immune Disorders Drug Targets, vol. 12, no. 2, pp. 148-158, 2012.

[3] J. D. Huber, "Diabetes, cognitive function, and the blood-brain barrier," Current Pharmaceutical Design, vol. 14, no. 16, pp. 1594-1600, 2008.

[4] T. Kuwashiro, H. Sugimori, T. Ago, J. Kuroda, M. Kamouchi, and T. Kitazono, "The impact of predisposing factors on long-term outcome after stroke in diabetic patients: the Fukuoka Stroke Registry," European Journal of Neurology, vol. 20, no. 6, pp. 921-927, 2013. 
[5] P. A. Wolf, R. B. D'Agostino, A. J. Belanger, and W. B. Kannel, "Probability of stroke: a risk profile from the Framingham Study," Stroke, vol. 22, no. 3, pp. 312-318, 1991.

[6] C. M. Burchfiel, J. D. Curb, B. L. Rodriguez, R. D. Abbott, D. Chiu, and K. Yano, "Glucose intolerance and 22-year stroke incidence. The Honolulu Heart Program," Stroke, vol. 25, no. 5, pp. 951-957, 1994.

[7] S. E. Megherbi, C. Milan, D. Minier et al., "Association between diabetes and stroke subtype on survival and functional outcome 3 months after stroke," Stroke, vol. 34, no. 3, pp. 688-694, 2003.

[8] P. G. Anthopoulos, S. J. Hamodrakas, and P. G. Bagos, “Apolipoprotein E polymorphisms and type 2 diabetes: a metaanalysis of 30 studies including 5423 cases and 8197 controls," Molecular Genetics and Metabolism, vol. 100, no. 3, pp. 283291, 2010.

[9] D. Chatzistefanidis, S. Giannopoulos, K. Spengos et al., “Apolipoprotein E polymorphisms and ischaemic stroke: a twocenter Greek study," European Journal of Neurology, vol. 21, no. 8, pp. 1083-1088, 2014.

[10] S. Das, S. Kaul, A. Jyothy, and A. Munshi, "Association of $A P O E$ (E2, E3 and E4) gene variants and lipid levels in ischemic stroke, its subtypes and hemorrhagic stroke in a South Indian population," Neuroscience Letters, vol. 628, pp. 136141, 2016.

[11] D. M. Hatters, C. A. Peters-Libeu, and K. H. Weisgraber, "Apolipoprotein E structure: insights into function," Trends in Biochemical Sciences, vol. 31, no. 8, pp. 445-454, 2006.

[12] S. Cosentino, N. Scarmeas, E. Helzner et al., "APOE 4 allele predicts faster cognitive decline in mild Alzheimer disease," Neurology, vol. 70, Issue 19, Part 2, pp. 1842-1849, 2008.

[13] L. Paternoster, N. A.. Martínez González, S. Lewis, and C. Sudlow, "Association between apolipoprotein E genotype and carotid intima-media thickness may suggest a specific effect on large artery atherothrombotic stroke," Stroke, vol. 39, no. 1, pp. 48-54, 2008.

[14] D. Seripa, G. D'Onofrio, F. Panza, L. Cascavilla, C. Masullo, and A. Pilotto, "The genetics of the human APOE polymorphism," Rejuvenation Research, vol. 14, no. 5, pp. 491-500, 2011.

[15] R. W. Mahley and S. C. Rall Jr., “APOLIPOPROTEINE: far more than a lipid transport protein," Annual Review of Genomics and Human Genetics, vol. 1, no. 1, pp. 507-537, 2000.

[16] V. van Giau, E. Bagyinszky, S. S. An, and S. Y. Kim, "Role of apolipoprotein E in neurodegenerative diseases," Neuropsychiatric Disease and Treatment, vol. 11, pp. 1723-1737, 2015.

[17] R. W. Mahley, "Apolipoprotein E: cholesterol transport protein with expanding role in cell biology," Science, vol. 240, no. 4852, pp. 622-630, 1988.

[18] L. Kopin and C. Lowenstein, "Dyslipidemia," Annals of Internal Medicine, vol. 167, no. 11, pp. ITC81-ITC96, 2017.

[19] A. American Diabetes, "Diagnosis and classification of diabetes mellitus," Diabetes Care, vol. 33, Suppl 1, pp. S62-S69, 2009.

[20] T. Almdal, H. Scharling, J. S. Jensen, and H. Vestergaard, "The independent effect of type 2 diabetes mellitus on ischemic heart disease, stroke, and death," Archives of Internal Medicine, vol. 164, no. 13, pp. 1422-1426, 2004.

[21] T. Jeerakathil, J. A. Johnson, S. H. Simpson, and S. R. Majumdar, "Short-term risk for stroke is doubled in persons with newly treated type 2 diabetes compared with persons without diabetes," Stroke, vol. 38, no. 6, pp. 1739-1743, 2007.

[22] B. N. Mankovsky and D. Ziegler, "Stroke in patients with diabetes mellitus," Diabetes/Metabolism Research and Reviews, vol. 20, no. 4, pp. 268-287, 2004.

[23] R. Chaudhary, A. Likidlilid, T. Peerapatdit et al., "Apolipoprotein E gene polymorphism: effects on plasma lipids and risk of type 2 diabetes and coronary artery disease," Cardiovascular Diabetology, vol. 11, no. 1, p. 36, 2012.

[24] L. K. Wei, A. Au, S. Menon et al., "Polymorphisms of MTHFR, eNOS, ACE, AGT, ApoE, PON1, PDE4D, and ischemic stroke: meta-analysis," Journal of Stroke and Cerebrovascular Diseases, vol. 26, no. 11, pp. 2482-2493, 2017.

[25] Q. Y. Wang, W. J. Wang, L. Wu, L. Liu, and L. Z. Han, "Metaanalysis of APOE $\varepsilon 2 / \varepsilon 3 / \varepsilon 4$ polymorphism and cerebral infarction," Journal of Neural Transmission (Vienna), vol. 120, no. 10, pp. 1479-1489, 2013.

[26] A. Kumar, P. Kumar, M. Prasad, S. Misra, A. Kishor Pandit, and K. Chakravarty, "Association between apolipoprotein $\varepsilon 4$ gene polymorphism and risk of ischemic stroke: a meta-analysis," Annals of Neurosciences, vol. 23, no. 2, pp. 113-121, 2016.

[27] J. Zhou, Y. L. Xue, Y. X. Guan, Y. D. Yang, S. B. Fu, and J. C. Zhang, "Association study of apolipoprotein e gene polymorphism and cerebral infarction in type 2 diabetic patients," $Y i$ Chuan, vol. 27, no. 1, pp. 35-38, 2005.

[28] C. H. Tseng, C. K. Chong, J. J. Sheu, T. H. Wu, and C. P. Tseng, "Prevalence and risk factors for stroke in type 2 diabetic patients in Taiwan: a cross-sectional survey of a national sample by telephone interview," Diabetic Medicine, vol. 22, no. 4, pp. 477-482, 2005.

[29] K. Al-Rubeaan, F. Al-Hussain, A. M. Youssef, S. N. Subhani, A. H. Al-Sharqawi, and H. M. Ibrahim, "Ischemic stroke and its risk factors in a registry-based large cross-sectional diabetic cohort in a country facing a diabetes epidemic," Journal Diabetes Research, vol. 2016, article 4132589, pp. 1-9, 2016. 